\title{
The Impact of New Features on Positioning Technology in LTE-A System
}

\author{
Zhang Bo, Du Yuanfeng, and Yang Dongkai \\ School of Electronic and Information Engineering, Beihang University, Beijing 100191, China \\ Correspondence should be addressed to Du Yuanfeng; yfdu1989@163.com
}

Received 15 August 2013; Accepted 24 February 2014

Academic Editor: David Taniar

Copyright (C) 2015 Zhang Bo et al. This is an open access article distributed under the Creative Commons Attribution License, which permits unrestricted use, distribution, and reproduction in any medium, provided the original work is properly cited.

\begin{abstract}
As the location based services develop, more and more researches have been focused on the positioning technologies in mobile networks. The long term evolution advanced (LTE-A) system, commercialized as the 4th generation (4G) mobile communication system, is based on the following key features: the orthogonal frequency division multiplexing (OFDM), the relay, the multiple input multiple outputs (MIMO), the carrier aggregation (CA), and the coordinated multipoint transmission and reception (CoMP). In this paper, the impact of these features on the existing positioning technology specified in the LTE-A standards is systematically investigated. Moreover, two approaches are proposed to take full advantage of these features in terms of positioning technologies and the key positioning parameters, including the reference signal time difference (RSTD) in the observed time difference of arrival (OTDOA) technology and user equipment receiving time subtracting transmitting time (UE Rx-Tx) in the enhanced cell identity (E-CID) technology.
\end{abstract}

\section{Introduction}

Location based services are highly demanding in various scenarios and global navigation satellite system (GNSS) has been developed for this purpose. In challenging environments, however, it is difficult to achieve accuracy. Supplementary network based positioning systems, such as the LTE-A systems, are then introduced $[1,2]$. To maximize positioning capabilities of these systems, many positioning technologies are proposed, such as assisted GNSS, OTDOA, and E-CID technologies. In this paper, we will focus on the latter two technologies.

The OTDOA technology is based on measurement of reference signal time difference (RSTD) from different base stations. The location of a UE can be obtained from the intersection point of hyperbolas (shown in Figure 1) [3]. Though this technology has been studied for a long time, it still has not been widely used in the existing wireless systems for small site detected numbers and low measurement accuracy. Later, the positioning reference signal (PRS) is specially specified in LTE-A system to improve the OTDOA performance. In [4-6], positioning methods based on the RSTD or TDOA have been proposed. The evaluation in [7] shows clearly the possibility of using 3GPP LTE measurements for indoor positioning. In [8], a method is carried out based on real measurement of OTDOA in the LTE $20 \mathrm{MHz}$ bandwidth using three base stations (BS) in realistic deployment. And one main conclusion is that the measured channel allows a positioning accuracy of $20 \mathrm{~m}$ and $63 \mathrm{~m}$ at the median and $95 \%$ level respectively. However, the results in the form researches are almost all in the general case and the accuracy of RSTD is guaranteed. The accuracy in the interference environment has not been investigated yet.

The E-CID method requires the angle of arrival (AOA) and time of arrival (TOA) measurements (shown in Figure 2). The positioning approach based on the TOA and AOA is introduced in $[9,10]$. However, the AOA is mainly based on the smart antenna and is hard to evaluate [11]. In this paper, only the accuracy of TOA is considered.

Many existing researches are about the positioning algorithms. However, besides the specific positioning algorithm, the measurements for positioning are also very important, such as RSTD and UE Rx-Tx, which are crucial since most methods are based on these measurements [12, 13]. How 


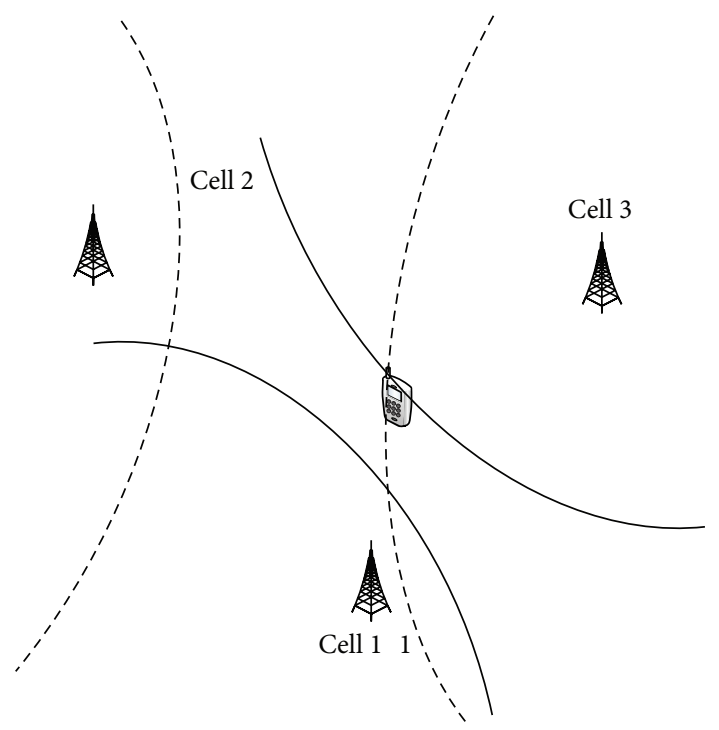

FIGURE 1: OTDOA positioning technology.

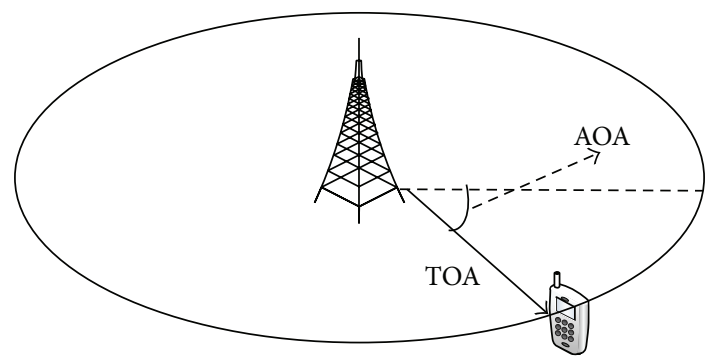

Figure 2: E-CID positioning technology.

to maintain their accuracy under interference is of great importance. Usually the interference on positioning can be introduced by the data transmission of base stations nearby [14]. Existing researches mainly focus on the intercell interference with data transmission [15]. To ensure the accuracy, the LTE-A standard has required that the PRS from each site is measured by a UE in different subframes without data transmission. In that case, there is no intercell interference but the interference of LPNs, such as relay, remote radio head (RRH), and femto play a nonnegligible impact. LPNs are operated for larger coverage area as mobile communication rapidly develops, which are introduced to improve throughput capacities of hot spots. The public LPNs can be controlled by the network, while the femtos are relatively independent.

Although the positioning technology has been widely used in personal location, vehicle navigation, advisory services, and other fields in the $2 \mathrm{G}$ and $3 \mathrm{G}$ systems, there are still many weaknesses. The low positioning accuracy, small service area, and long positioning delay have impacted the quality of location service. With the development of LTE-A system, high information speed and more colorful multimedia services can be achieved. It is evident that the strength of the positioning signal in LTE-A system will be significantly improved by the new technologies and features. On the other hand, the interference on positioning measurement will also be increased. Due to more detected base stations, small modification of the existing positioning technologies is also needed. Thus, it is important to study the impact of LTE-A system on positioning technology deeply.

This paper proposed a novel direction to improve the positioning technology in the LTE-A systems. Instead of improving the positioning algorithms in the normal researches, the main objective of this paper is to investigate the impact of new features on positioning technology in LTEA systems and how to take advantage of them, such as the OTDOA and E-CID. The evaluation process is based on the accuracy requirement specified in 3GPP LTE-A standards. Our contributions are as follows:

(a) establishing system model in detail to detect the time of signal arrival for RSTD and UE Rx-Tx measurement;

(b) quantifying the impact of LPNs on measurement accuracy and proposing approaches to improve the accuracy;

(c) modifying the existing positioning technologies based on the CA and CoMP features.

The rest of this paper is organized as follows. In Section 2, the system model for RSTD and UE Rx-Tx is introduced. Thereafter, accuracy performance is studied under the interference of LPNs and new approaches are proposed to recover the accuracy requirement in Section 3. Minor modification of the existing positioning technologies is proposed in Section 4. Conclusions are drawn in Section 5.

\section{System Model for Positioning Measurement}

Multipath and nonline of sight (NLOS) are widespread in the city environment, which results in poor positioning accuracy [16]. The OFDM technology used in LTE-A system has advantage of antifading and anti-interference, which enhances the receiving and identification of the positioning signal, and provides good foundation for the improvement of positioning accuracy $[2,17,18]$. Only the line of sight (LOS) scenario with multipath interference is considered in this section.

A method for detecting the RSTD accuracy has been briefly described based on the discussion of 3GPP reports [19]. To study accuracy of positioning measurement, the system model based on the method for detecting the time of signal arrival in baseline scenario is established first. Measurement of RSTD and UE Rx-Tx can be described by the same system model except a different resource mapping technique. More specifically, PRS resource mapping is used for RSTD and cell-specific reference signal (CRS) resource mapping is used for UE Rx-Tx.

According to the 3rd Generation Partnership Project (3GPP) Ts36.211 [20], basic scenarios for positioning measurement are presented in Figure 3. In Figure 3(a), the PRS is sent out from two eNobes to the UE with the same distance and the RSTD is supposed to be zero. In Figure 3(b), the CRS is sent out from the serving eNobe to the UE and the real travel time is known. 


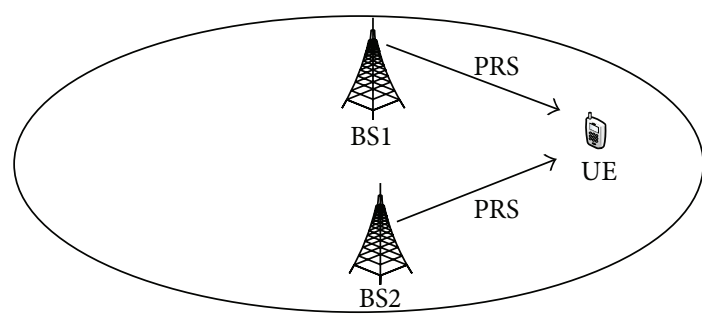

(a) Scenario for PRS

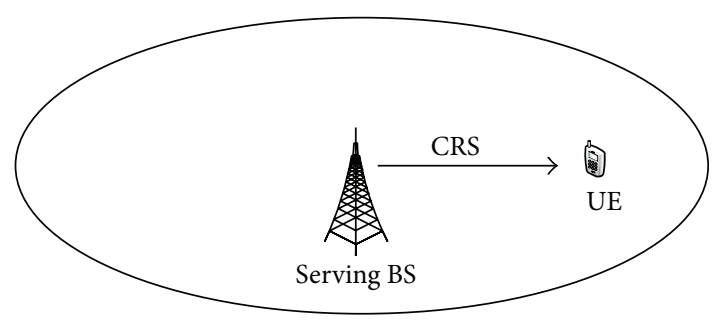

(b) Scenario for CRS

FIgURe 3: Positioning measurement scenarios.

The common system model is presented in Figure 4. In step 1, the signal sequence $r_{l, n_{\mathrm{s}}}(m)$ is constructed as follows:

$$
\begin{aligned}
& r_{l, n_{\mathrm{s}}}(m)=\frac{1}{\sqrt{2}}(1-2 \cdot c(2 m)) \\
& \quad+j \frac{1}{\sqrt{2}}(1-2 \cdot c(2 m+1)), \\
& \quad m=0,1, \ldots, 2 N_{\mathrm{RB}}^{\max , \mathrm{DL}}-1,
\end{aligned}
$$

where $l$ is the orthogonal frequency division multiplexing (OFDM) symbol number in one slot, $n_{s}$ is the slot number in one radio frame, $j$ is the imaginary unit, and $N_{\mathrm{RB}}^{\max , \mathrm{DL}}$ is the number of resource blocks in the downlink bandwidth. The pseudorandom sequence $c(i)$ here can be initialized at the beginning of each OFDM symbol with $c_{\text {init }}$ given by

$$
\begin{aligned}
c_{\text {init }}= & 2^{10} \cdot\left(7 \cdot\left(n_{\mathrm{s}}+1\right)+l+1\right) \cdot\left(2 \cdot N_{\mathrm{ID}}^{\text {cell }}+1\right)+2 \\
& \cdot N_{\mathrm{ID}}^{\text {cell }}+1,
\end{aligned}
$$

where $N_{\text {ID }}^{\text {cell }}$ is the identification of a cell.

In step 2, the signal sequence is mapped into the timefrequency resource block according to structures specified in the standards. This is done on a uniform mesh over the timefrequency domain, where time and frequency are employed as horizontal and vertical axes, respectively. This sequence is then modulated and processed by the inverse fast Fourier transform (IFFT). Adding the cyclic prefix (CP), the time domain signal is obtained.

In step 3, the signal is transmitted through a channel, which is described by the additive white Gaussian noise (AWGN) model or the extended typical urban (ETU) model. The output signal is obtained after combining with an additive white noise.

After receiving the signal, propagation time of the signal between a UE and a site can be measured through estimating the first arrived path [21]. The basic principle is that analyzing the correlation between the received signal and a local signal (or the transmitted signal, equivalently). A signal correlation spectrum can be generated when the shifting time $(x)$ and correlation $(y)$ are employed as $x$ - and $y$-axis, respectively. More precisely, the signal bandwidth $10 \mathrm{MHz}$ is divided into 1024 chips, where a chip is the basic unit of a signal in the time domain, and the signal correlation is obtained when shifting the received signal by chips. One chip in $10 \mathrm{MHz}$ and

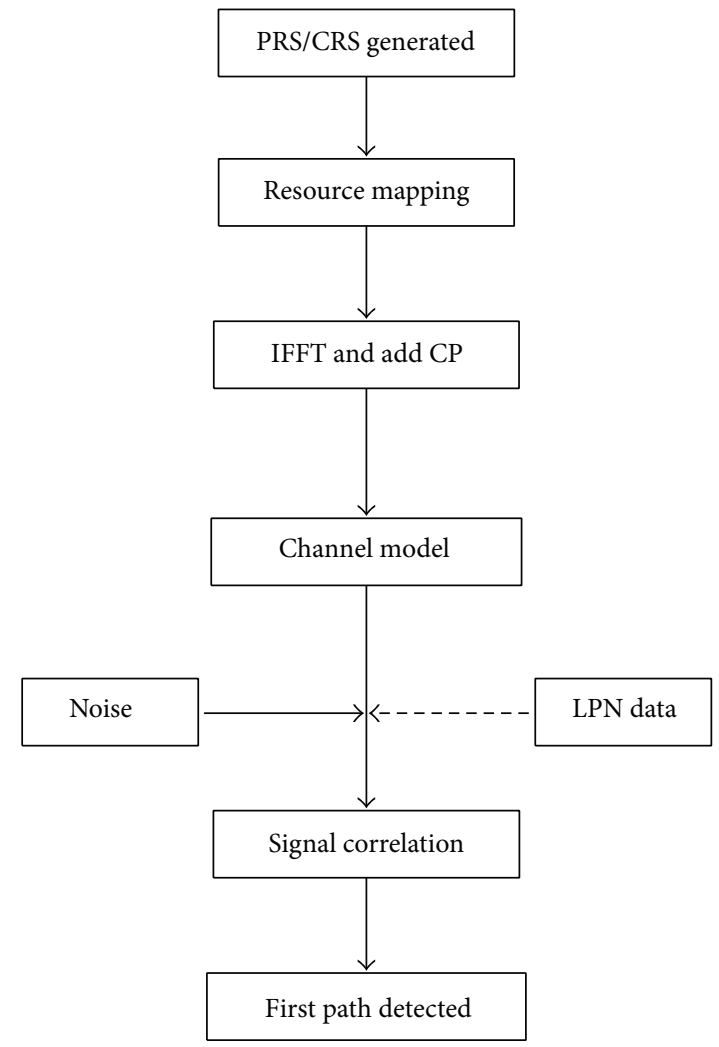

Figure 4: System model.

1.4 MHz system equals $2 \mathrm{Ts}$ and $16 \mathrm{Ts}$, respectively, with the time granularity Ts standing for $32.5 \mathrm{~ns}$.

The first arrived path is defined as the minimum chip satisfying

$$
\min _{x}\left\{x \mid y(x) \in\left[y_{l b}, y_{\text {peak }}\right]\right\} \cap\left\{x \mid y(x) \geq y_{\text {gate }}\right\} \text {. }
$$

Here $y_{\text {peak }}, y_{\text {gate }}$, and $y_{l b}$ are the peak values of the signal correlation, the given noise gate, and the lower bound of the searching interval, respectively. In this simulation,

$$
y_{l b}=y_{\text {peak }}-\alpha
$$

where $\alpha$ is the parameter to avoid the false alarm problem and an empirical strategy is proposed to choose $y_{\text {gate }}=5.1 \mathrm{~dB}$ and $\alpha=8 \mathrm{~dB}$ for $1 \%$ false alarm probability in [19]. 
In the LTE-A system, MIMO is widely used and the deployment of antennas typically involves one transmitting antenna and two receiving antennas, which produce two signal correlation spectrums. The correlation spectrum used for the detection of the first path is the average of the above two spectrums.

So far, the model only takes into account the baseline scenario. Difference between the measured result time detect and the actual value time $e_{\text {true }}$ is considered as measurement accuracy Error $r_{\text {measure }}$ :

$$
\text { Error }_{\text {measure }}=\text { time }_{\text {true }}-\text { time }_{\text {detect }} \text {. }
$$

To mimic the interference induced by a LPN, another signal containing random data sequences Data ${ }_{L P N}$ over the whole bandwidth is generated and added to the initial transmitting signal:

$$
\operatorname{Data}_{\mathrm{LPN}}=10^{\mathrm{SNR}_{\mathrm{LPN}} / 10} * D,
$$

where $\mathrm{SNR}_{\mathrm{LPN}}$ is the signal-to-noise ratio (SNR) of LPN. $D$ is the random binary sequence after binary phase shift keying (BPSK) modulation.

\section{Impact of LPNs on Positioning Measurement}

The basic function of the LPNs is to retransmit the signal from the BS or a UE, with the purpose of improving the coverage of high data rates and providing temporary coverage to fill shadow regions. With the development of mobile networks, the LPNs have been widely used and become an important feature of LTE-A system. In this section, the research of the impact of LPNs on positioning measurement includes the following RSTD and UE Rx-Tx two parts. The detailed system simulation model for detecting the first path arrived to obtain the RSTD and UE Rx-Tx measurement has been proposed in Section 2. To compare with the accuracy requirement in the 3GPP LTE standard, a lot of experiment results are obtained in various scenarios. Two approaches, furthermore, are proposed to improve the accuracy of these parameters in order to access the positioning requirement of LTE-A system with excellent simulation performance.

3.1. Impact of RSTD under Interference of LPNs. As specified in 3GPP 36.133 [22], the accuracy requirement of RSTD is $15 \mathrm{Ts}$ in $1.4 \mathrm{MHz}$ and $5 \mathrm{Ts}$ in $10 \mathrm{MHz}$, where time granularity Ts stands for $32.5 \mathrm{~ns}$ ( $2 \mathrm{Ts}=1 \mathrm{chip})$. If the margin and actual measurement errors are excluded, the suitable requirement is around 1 Ts.

In the conventional OTDOA technology, LPNs are not considered as positioning nodes [23]. Therefore, data transmission through LPNs may introduce strong interference. In Figure 5, the nearer the UE is from the LPN, the more the interference will be introduced in the measurement of RSTD. We should research to answer the following questions: what is the interference degree from the LPNs on RSTD measurements and how to eliminate the interference?

Based on the system model proposed above, the following detailed parameters are set for the simulation in Table 1 . As

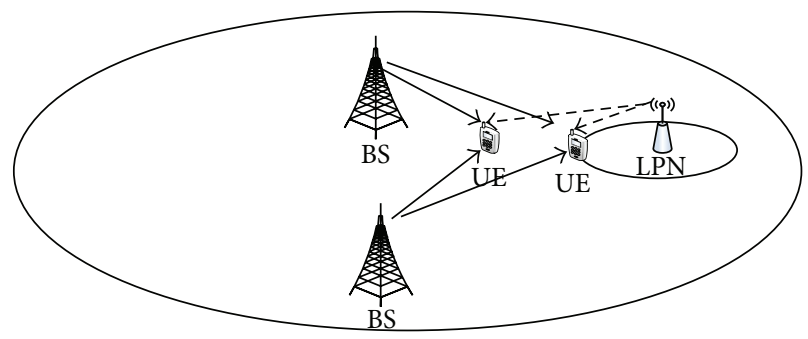

$\longrightarrow$ PRS

$--\rightarrow$ Interference

FIGURE 5: Interference of LPN on RSTD.

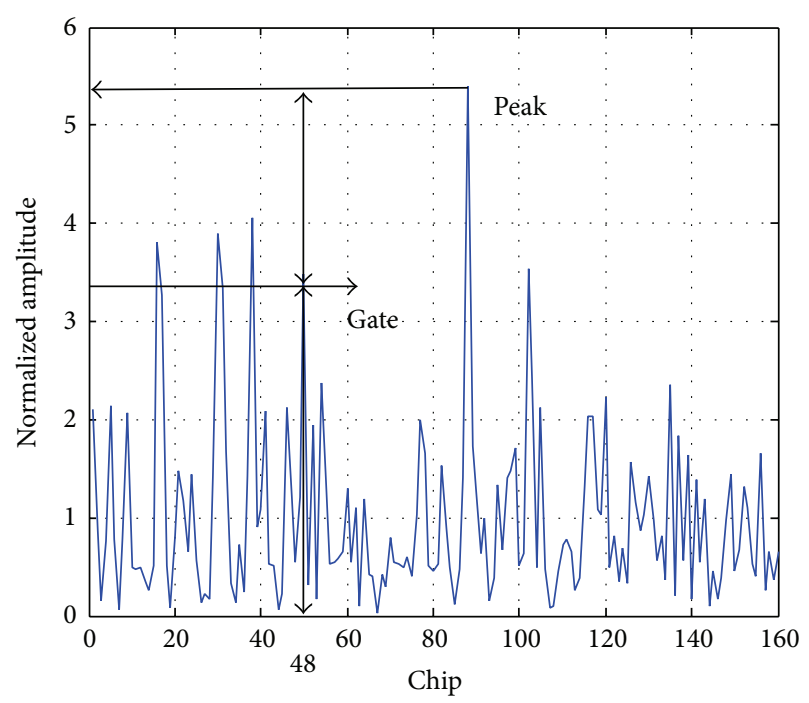

FIGURE 6: Signal correlation in $10 \mathrm{MHz}$ with LPN.

shown in Figure 6, if a UE is close to a LPN during the positioning service, the detection of the real first arrived path would be difficult for many correlation peaks. As a result, the detected result is the 24.1th chip while the true time is in the 15th chip, with the measurement error of 18.2 Ts (9.1 chips). Thus the additional error introduced by LPNs is too large to be ignored.

To analyze the interference degree of a LPN, signal-tonoise ratio (SNR) of the main positioning site is set as $-13 \mathrm{~dB}$ according to the minimum detecting threshold for PRS, and SNR of the LPN varies from $-12 \mathrm{~dB}$ to $6 \mathrm{~dB}$. Figures 7 and 8 show that RSTD error is an increasing function of SNR. The error increases dramatically when SNR is greater than $0 \mathrm{~dB}$ in $10 \mathrm{MHz}$ and $1.4 \mathrm{MHz}$ and exceeds the accuracy requirement.

3.2. Approach Proposed for RSTD Measurement. To solve this problem, the following approach is proposed in Figure 9 as follows.

(a) For a public LPN, set a threshold for SNR. If the interference degree detected by the UE is greater than the threshold $\left(\mathrm{SNR}_{\mathrm{LPN}} \geq 0 \mathrm{~dB}\right)$, the interference should be eliminated; otherwise, no action is required. For the former 
TABLE 1: RSTD simulation parameters.

\begin{tabular}{lcc}
\hline Parameter & Configuration & Remarks \\
\hline Measurement bandwidth & $1.4 \mathrm{MHz}, 10 \mathrm{MHz}$ & Two cases \\
Measurement resource block & $6 \mathrm{RB}, 50 \mathrm{RB}$ & Two cases \\
Measurement period & $200 \mathrm{~ms}$ & \\
Transmitting antenna number & 1 & The two antennas are not related \\
Receiving antenna number & 2 & Two cases \\
Channel & AWGN & The interference from other cells is included in the noise \\
CP length & Normal CP \\
Frequency & $2.0 \mathrm{GHz}$ & \\
SNR of positioning cell & $(-3 \mathrm{~dB},-13 \mathrm{~dB})$ & \\
SNR of LPN & {$[-\propto, 6 \mathrm{~dB}]$} & \\
\hline
\end{tabular}

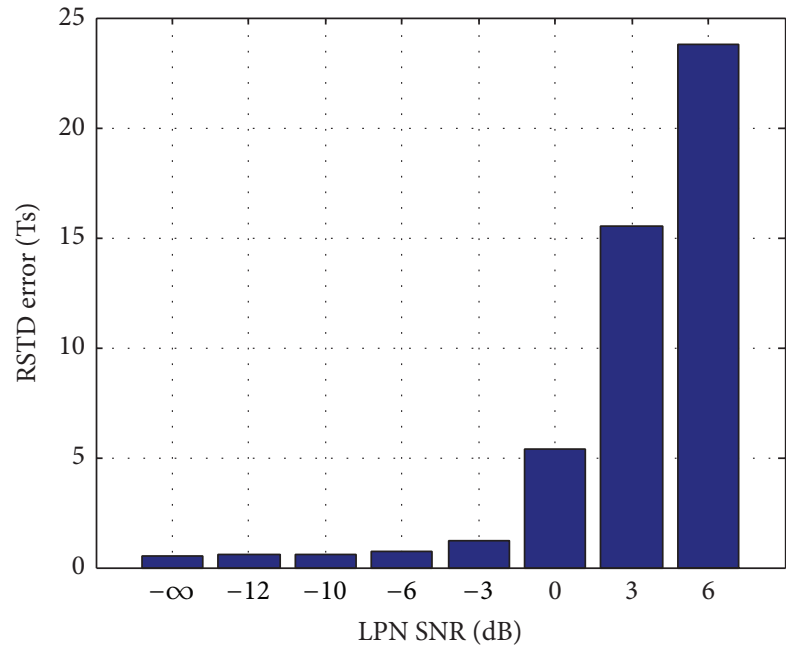

FIGURE 7: RSTD error as a function of LPN SNR in $10 \mathrm{MHz}$.

case, if this LPN can transmit PRS, it is then used as a temporary positioning node; otherwise, lower data transmitting power or terminate data transmission is used to reduce the interference.

Figures 10 and 11 show the results of the above approach under interference. The correlation peak in Figure 4 is quite obvious compared with Figure 6, which makes the detection of the first arrived path much easier. And the detected result is just around the 15th chip, which is the true time. The cumulative distribution function (CDF) of RSTD accuracy in Figure 11 shows that $90 \%$ of the RSTD error is smaller than 1 Ts and is consistent with the standard. By taking interfering signals into account, the proposed approach is effective to ensure the accuracy requirement of RSTD.

(b) For a private LPN, its coverage area is always less than $40 \mathrm{~m}$. UEs suffering strong interference from this LPN can use the location of this LPN as the positioning result which satisfies the Federal Communications Commission accuracy requirement.

3.3. Impact of UE Rx-Tx under Interference of LPNs. For E-CID positioning technology, the distance between the

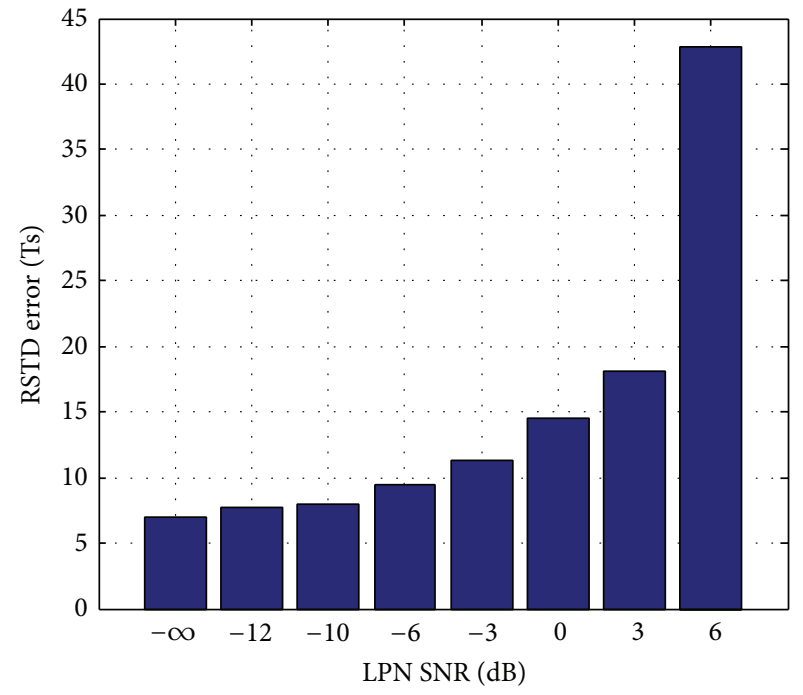

FIGURE 8: RSTD error as a function of LPN SNR in $1.4 \mathrm{MHz}$.

serving BS and UE is indicated by time of arrival (TOA); the detailed measurement process of TOA is shown in Figure 12 as follows:

T1: time of transmitting uplink signal from UE to BS;

T2: time of transmitting downlink signal from UE to BS;

T3: true time of uplink signal arriving at BS;

T4: arriving time of uplink signal measured by BS (with error);

T5: true time of downlink signal arriving at UE;

T6: arriving time of downlink signal measured by UE (with error).

Obviously, $\mathrm{TA}=(\mathrm{T} 3-\mathrm{T} 2)+(\mathrm{T} 5-\mathrm{T} 1)=(\mathrm{T} 3-\mathrm{T} 1)+(\mathrm{T} 5-$ T2) $=2 *$ TOA. When the measurement error introduced by BS (T4 - T3) is ignored, the error on the UE side (T6 - T5) is considered as the main factor of TOA error and denoted by the UE Rx-Tx error [10].

The scenario of LPN interference on UE Rx-Tx is shown in Figure 13. The accuracy requirement of UE Rx-Tx is $10 \mathrm{Ts}$ in $10 \mathrm{MHz}$ and $20 \mathrm{Ts}$ in $1.4 \mathrm{MHz}$, and minimum SNR of a CRS 
TABLE 2: UE Rx-Tx simulation parameters.

\begin{tabular}{lcc}
\hline Parameter & Configuration & Remarks \\
\hline Measurement bandwidth & $1.4 \mathrm{MHz}, 10 \mathrm{MHz}$ & Two cases \\
Measurement resource block & $6 \mathrm{RB}, 50 \mathrm{RB}$ & Two cases \\
Measurement period & $200 \mathrm{~ms}$ & Coherent average of each sample \\
Samples & 5 & The two antennas are not related \\
L3 filter & Not used & Two cases \\
Transmitting antenna number & 1 & The interference from other cells is included in the noise \\
Receiving antenna number & 2 & AWGN, ETU70 \\
Channel & Normal CP & $2.0 \mathrm{GHz}$ \\
Frequency & $-3 \mathrm{~dB}$ & \\
SNR of serving cell &
\end{tabular}

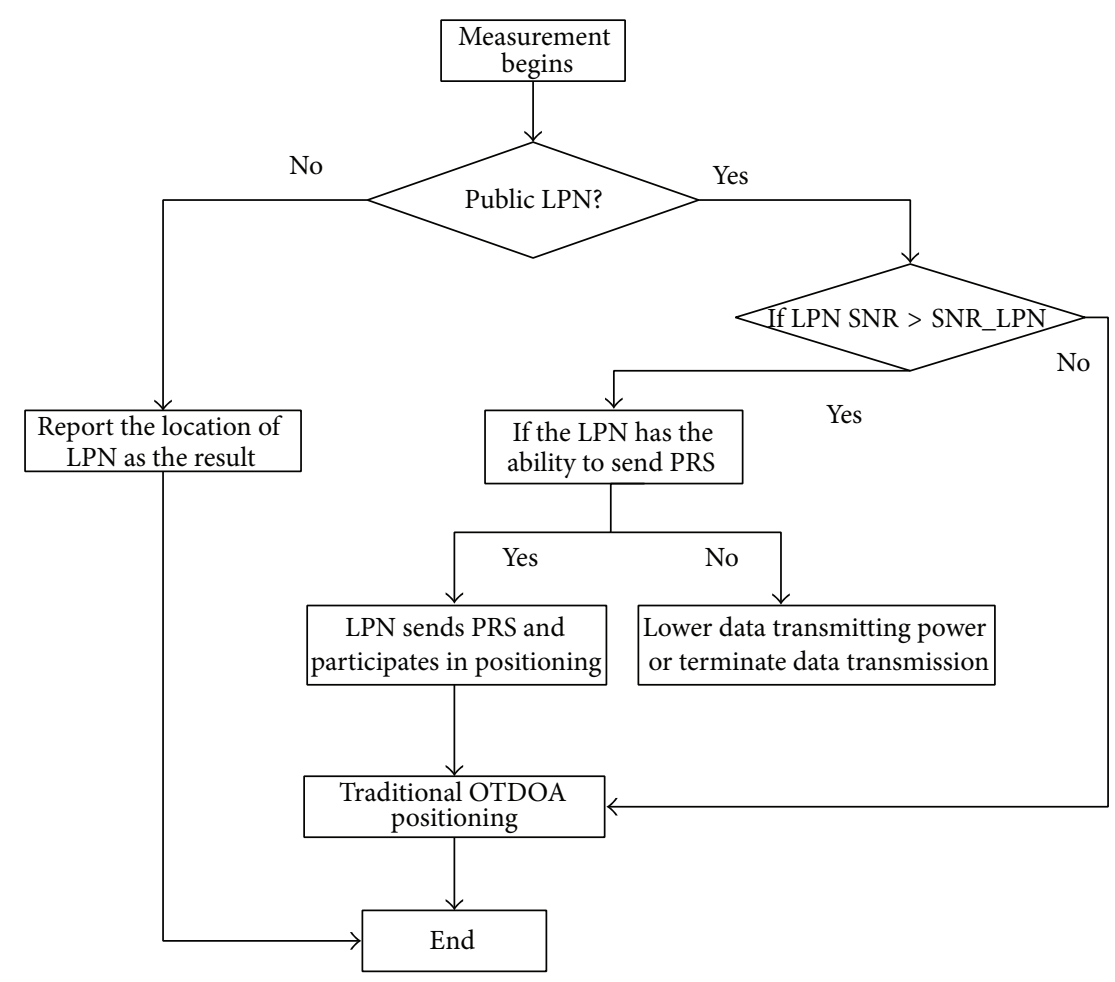

FIGURE 9: Approach proposed for RSTD measurement under the LPN interference.

from a serving site is $-3 \mathrm{~dB}$ [9]. The typical interference of a $\mathrm{LPN}$ is $-1 \mathrm{~dB}$, and the other detailed parameters are set for the simulation in Table 2.

The approach in Section 3.2, designed for RSTD, can also be used here to improve the accuracy of Rx-Tx [24]. However, the data transmitting service of a LPN will be affected if strategy (a) is used.

Therefore, the method of measuring on the multicastbroadcast single frequency network (MBSFN) subframes is proposed, which works for both public and private LPNs [25]. The collision case is named if CRS pilots of the measuring cell and the aggressive LPN are the same; otherwise, it is called noncollision case. Performance of this method under different conditions is listed in Figures 14 and 15 where cases 1,2 , and 3 stand for interference (non-MBSFN), MBSFN approach without collision, and MBSFN approach with collision, respectively. And more simulation results are collected in Table 3, in which the $90 \%$ value of the CDF is presented. In the wideband situation $(10 \mathrm{MHz})$, as the $\mathrm{UE}$ is usually near the serving cell, the interference is weak compared with the signal strength of the serving cell. Thus, the measurement of UE Rx-Tx is less affected, and the improvement of MBSFN approaches is not obvious. However, in the narrowband situation $(1.4 \mathrm{MHz})$, the interference is strong compared with the signal strength. Hence, MBSFN approaches improve the accuracy in a significant way. 
TABLE 3: Accuracy of UE Rx-Tx.

\begin{tabular}{lccc}
\hline Channel model & & AWGN & Case 3 \\
\hline Bandwidth & Case 1 & Case 2 & $-6.05 \sim-4.92$ \\
$1.4 \mathrm{MHz}(\mathrm{Ts})$ & $-7.95 \sim-5.36$ & $-5.2 \sim-4.44$ & $-0.56 \sim-0.53$ \\
$10 \mathrm{MHz}$ (Ts) & $-0.57 \sim-0.54$ & $-0.55 \sim-0.53$ & Case 3 \\
\hline Channel model & & ETU70 & $-6.75 \sim-4.46$ \\
\hline Bandwidth & Case 1 & Case 2 & $-0.22 \sim 5.4$ \\
$1.4 \mathrm{MHz}(\mathrm{Ts})$ & $-11.59 \sim-4.89$ & $-6.45 \sim-4.45$ & $-0.2 \sim 5.4$ \\
$10 \mathrm{MHz}(\mathrm{Ts})$ & $-0.29 \sim 5.4$ & & - \\
\hline
\end{tabular}

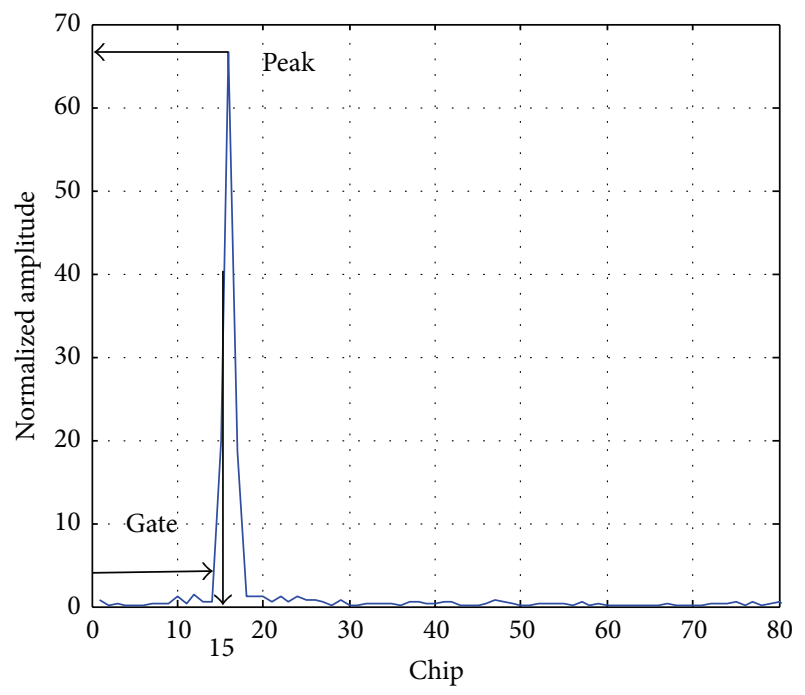

FIGURE 10: Signal correlation in $10 \mathrm{MHz}$.

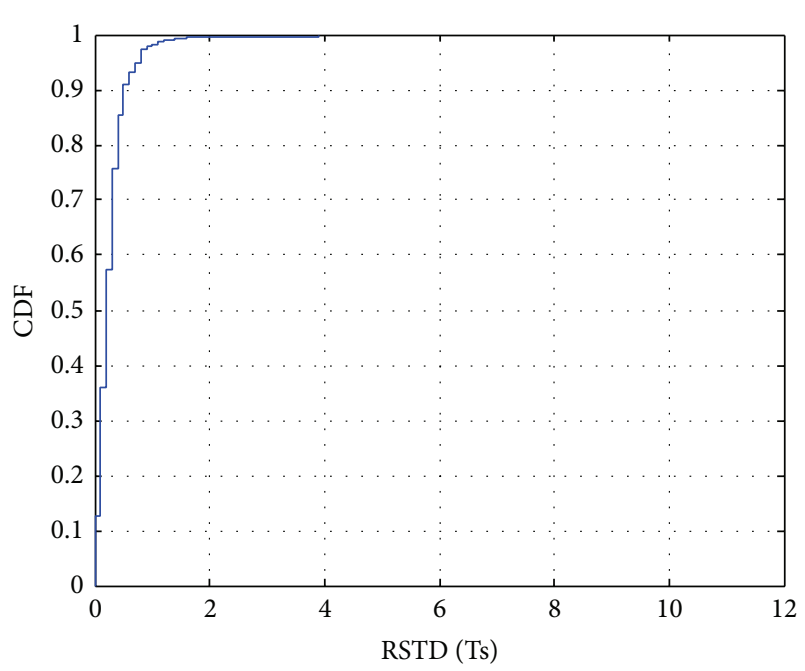

FIGURE 11: CDF of RSTD accuracy in $10 \mathrm{MHz}$ AWGN.

\section{Modification of Positioning Technology Based on CA and CoMP}

4.1. Impact of CA Technology on Positioning. In order to satisfy the highest rate in LTE-A system, the bandwidth

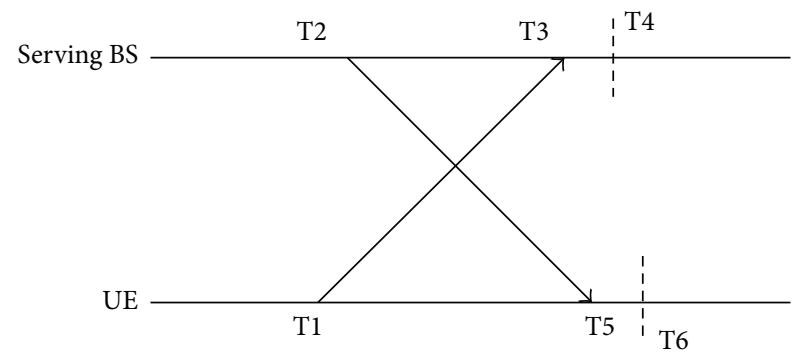

FIgURE 12: Timing relationship of downlink and uplink signal.

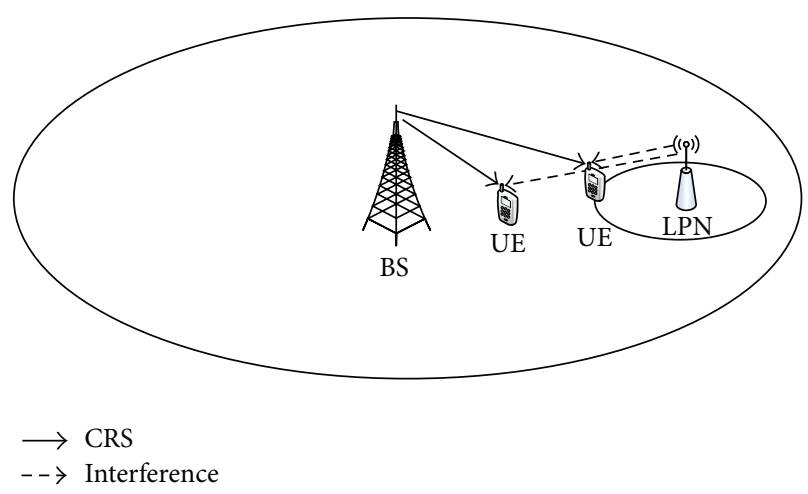

Figure 13: Interference of LPN on UE Rx-Tx.

should be as large as $100 \mathrm{MHz}$. Thus, the carrier aggregation (CA) technology is proposed in LTE-A system, which is used to combine the separate bandwidth together with different frequency carriers. In the CA scenario, each base station can allocate up to eight carriers to the user and the signal quality on each carrier varies. The PRS would be measured on one arbitrary carrier based on the positioning procedure in the LTE-A standards, which is not reasonable. The signal quality of each carrier should be measured before detecting the PRS and the one with the highest SNR is chosen for the positioning measurement. The improved positioning scheme is shown in Figure 16, in which the MMSE (mobility management equipment) and SMLC (senior management location center) are models. When UE sends the message of positioning ability response to SMLC, the proposed carrier quality response signaling containing quality information of each carrier for 


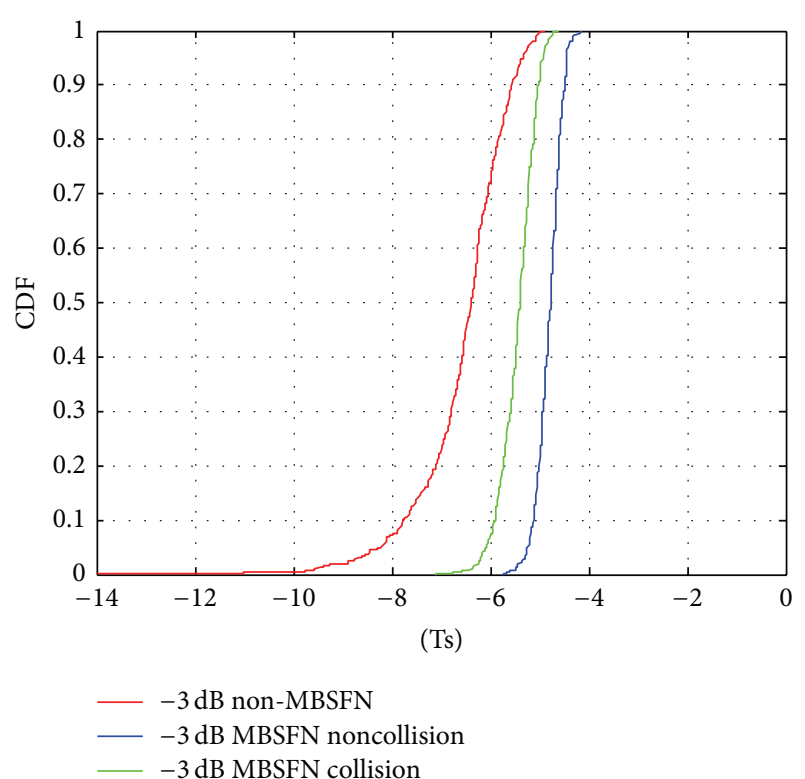

FIGURE 14: UE Rx-Tx accuracy in three cases with $1.4 \mathrm{MHz}$ and AWGN.

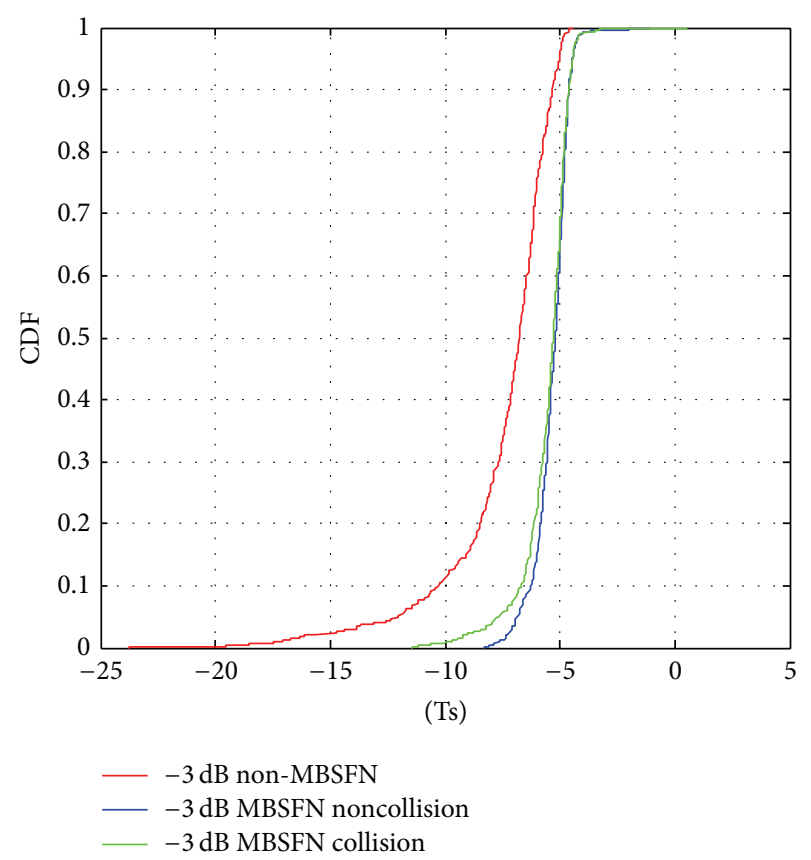

Figure 15: UE Rx-Tx accuracy in three cases with $1.4 \mathrm{MHz}$ and ETU70.

carrier selection should also been transmitted. The comparison in Table 4 shows that the improvement is obvious, especially in the three-BS case.

4.2. Impact of CoMP Feature on Positioning. In the LTE-A system, the CoMP feature is introduced to reduce intercell interference and improve the spectral efficiency for the edge UE. The feature provides several serving BS for the UE instead of a unique BS in the previous. Thus, existing E-CID

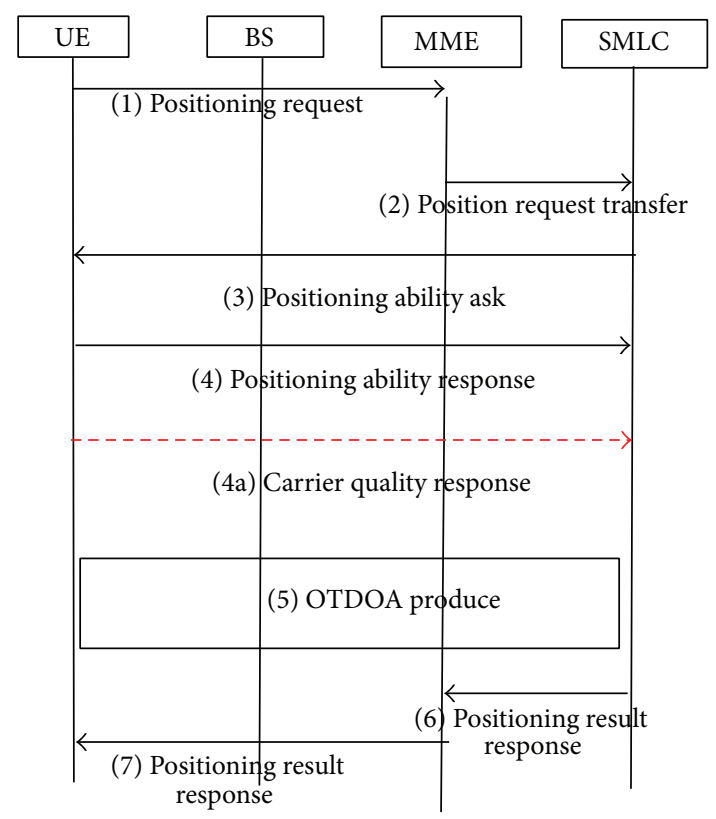

FIGURE 16: New workflow of OTDOA method.

positioning method can be improved based on the additional information from CoMP serving BSs. As the simulation above indicates, the accuracy of time of arrival (TOA) can be guaranteed in a small range. However, due to the multipath effects and limitations of hardware device, the angle of arrived (AOA) measurement has great error. Thus, the positioning result of E-CID is always ranging from 200 to 1000 meters. When the CoMP feature is introduced, the UE can perform TOA and AOA measurements from all the BSs in the CoMP set together. The positioning result can be considered in the crossover regions of several track rounds, which are generated with the location of BS as the circle center and the TOA as the radius. The inaccuracy AOA measurement is used as the auxiliary information to decrease the candidate regions (see Figure 17).

The formulas are as follows:

$$
\begin{array}{r}
\sqrt{\left(x_{\mathrm{ue}}-x_{i}\right)^{2}+\left(y_{\mathrm{ue}}-y_{i}\right)^{2}}=\mathrm{TOA}_{i} * c \\
\tan \left(\mathrm{AOA}_{i}\right)=\frac{y_{\mathrm{ue}}-y_{i}}{x_{\mathrm{ue}}-x_{i}} \\
i=1,2, \ldots, N, N
\end{array}
$$

where $N$ is the number of the CoMP set. $\left(x_{\mathrm{ue}}, y_{\mathrm{ue}}\right)$ is the location of the UE, and $\left(x_{i}, y_{i}\right)$ is the location of the $i$ th serving $\mathrm{BS}$. $\mathrm{TOA}_{i}$ and $\mathrm{AOA}_{i}$ are the measurements from the $i$ th serving $\mathrm{BS}$.

\section{Conclusions and Future Directions}

We have systematically investigated key parameters for positioning in LTE-A system under the interference of LPNs. 
TABLE 4: Compared result of the proposed scheme.

\begin{tabular}{lcccc}
\hline \multirow{2}{*}{ Positioning error } & \multicolumn{2}{c}{ Three-BS case (probability) } & \multicolumn{2}{c}{ Four-BS case (probability) } \\
& Random carrier & Proposed method & Random carrier & Proposed method \\
\hline$<50 \mathrm{~m}$ & $64.4 \%$ & $69.2 \%$ & $90 \%$ & $92 \%$ \\
$<150 \mathrm{~m}$ & $74 \%$ & $77.4 \%$ & $96.1 \%$ & $97.8 \%$ \\
\hline
\end{tabular}

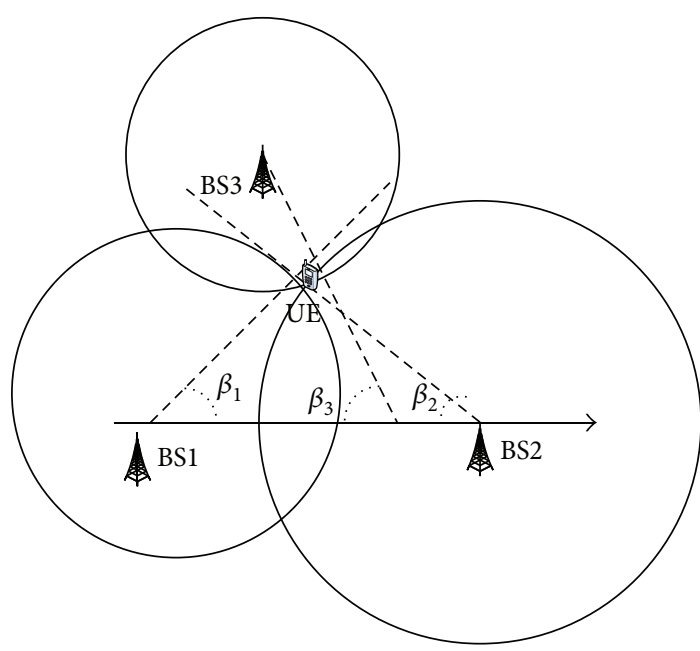

Figure 17: Modification for E-CID method in CoMP scenario.

To achieve accuracy requirement, two approaches are proposed with excellent simulation performance. Moreover, modifications are proposed to improve existing positioning methods especially in the CA and CoMP scenarios. Extensive simulations show that proposed approaches are effective. Since observation measurements from experimental campaigns can provide realistic strengths of signal interference introduced by LPNs, to what extent our approaches are applicable will be an interesting topic.

As the positioning accuracy is greatly affected by the measurements of signals, such as RSTD and TOA, further researches will be focused on the approaches of ensuring the measurements in various real environments beyond the improvement of positioning algorithms. The benefits for positioning introduced by CA, MIMO, and CoMP technologies should be investigated more deeply. Moreover, as the development of the mobile communication system, new framework specified for positioning should be researched.

\section{Conflict of Interests}

There is no conflict of interests.

\section{Acknowledgments}

The research work was supported by National High-Tech Research and Development Program of China (863 Program) under Grant no. 2013AA12A201 and Doctor Innovative Research Fund of Beihang University.

\section{References}

[1] A. Dammann, E. Staudinger, S. Sand, and C. Gentner, "Joint GNSS and 3GPP-LTE based positioning in outdoor-to-indoor environments-performance evaluation and verification," in Proceedings of the 24th International Technical Meeting of the Satellite Division of the Institute of Navigation (ION GNSS '11), pp. 3587-3595, September 2011.

[2] C. Gentner, S. Sand, and A. Dammann, "OFDM indoor positioning based on TDOAs: performance analysis and experimental results," in Proceedings of the International Conference on Localization and GNSS (ICL-GNSS '12), Starnberg, Germany, June 2012.

[3] Y. T. Chan and K. C. Ho, "Simple and efficient estimator for hyperbolic location," IEEE Transactions on Signal Processing, vol. 42, no. 8, pp. 1905-1915, 1994.

[4] K. Yang, J. An, X. Bu, and G. Sun, "Constrained total leastsquares location algorithm using time-difference-of-arrival measurements," IEEE Transactions on Vehicular Technology, vol. 59, no. 3, pp. 1558-1562, 2010.

[5] R. Kaune, J. Horst, and W. Koch, "Accuracy analysis for TDOA localization in sensor networks," in Proceedings of the IEEE 14th International Conference on Information Fusion (FUSION '11), Chicago, Ill, USA, July 2011.

[6] W. Guo and S. Wang, "Interference-aware self-deploying Femto-cell," IEEE Wireless Communications Letters, vol. 1, no. 6, pp. 609-612, 2012.

[7] J. Medbo, I. Siomina, A. Kangas, and J. Furuskog, "Propagation channel impact on LTE positioning accuracy-a study based on real measurements of observed time difference of arrival," in Proceedings of the IEEE 20th Personal, Indoor and Mobile Radio Communications Symposium (PIMRC '09), pp. 2213-2217, IEEE, Tokyo, Japan, September 2009.

[8] C. Zhu, "High accuracy multi-link synchronization in LTE: applications in localization," in Proceedings of the 16th IEEE Mediterranean Electrotechnical Conference (MELECON '12), pp. 908-913, March 2012.

[9] C. Gentner, E. Muñoz, M. Khider, E. Staudinger, S. Sand, and A. Dammann, "Particle filter based positioning with 3GPP-LTE in indoor environments," in Proceedings of the IEEE/ION Position, Location and Navigation Symposium (PLANS '12), pp. 301-308, April 2012.

[10] S. Al-Jazzar, M. Ghogho, and D. McLernon, "A joint TOA/AOA constrained minimization method for locating wireless devices in non-line-of-sight environment," IEEE Transactions on Vehicular Technology, vol. 58, no. 1, pp. 468-472, 2009.

[11] T. Wigren, "Adaptive enhanced cell-ID fingerprinting localization by clustering of precise position measurements," IEEE Transactions on Vehicular Technology, vol. 56, no. 5, pp. 31993209, 2007.

[12] J. Kim, S. Kim, N. Y. Kim, J. Kang, Y. Kim, and K.-T. Nam, "A novel location finding system for 3GPP LTE," in Proceedings of 
the 20th IEEE Personal, Indoor and Mobile Radio Communications Symposium (PIMRC '09), pp. 3213-3217, Tokyo, Japan, September 2009.

[13] T. Wigren, I. Siomina, and M. Anderson, "Estimation of prior positioning method performance in LTE," in Proceedings of the IEEE 22nd International Symposium on Personal, Indoor and Mobile Radio Communications (PIMRC '11), pp. 1279-1283, IEEE, Toronto, Canada, September 2011.

[14] G. Boudreau, J. Panicker, N. Guo, R. Chang, N. Wang, and S. Vrzic, "Interference coordination and cancellation for $4 \mathrm{G}$ networks," IEEE Communications Magazine, vol. 47, no. 4, pp. 74-81, 2009.

[15] J. A. Del Peral-Rosado, J. A. Lopez-Salcedo, G. Seco-Granados, F. Zanier, and M. Crisci, "Achievable localization accuracy of the positioning reference signal of 3GPP LTE," in Proceedings of the International Conference on Localization and GNSS (ICL-GNSS '12), pp. 1-6, Starnberg, Germany, June 2012.

[16] J. Medbo, I. Siomina, and A. Kangas, "A propagation channel impact on LTE positioning accuracy," in Proceedings of the Personal, Indoor and Mobile Radio Communications (PIMRC '09), pp. 66-68, 2009.

[17] D. Serant, O. Julien, L. Ries, P. Thevenon, and M. Dervin, The Digital TV Case: Positioning Using Signals-of-Opportunity based on OFDM Modulation, Inside GNSS, Eugene, Ore, USA, 2011.

[18] T. E. Sorensen, P. E. Mogensen, and F. Frederiksen, "Extension of the ITU channel models for wideband (OFDM) systems," in Proceedings of the 62nd IEEE Vehicular Technology Conference (VTC '05), vol. 1, pp. 392-396, IEEE, September 2005.

[19] 3GPP, "Proposed system simulation assumptions for OTDOA positioning," Ericsson R4-093039, ST-Ericson, 2009.

[20] 3GPP; TR 36211, Physical Channels and Modulation, Version 11.1.0, 2012.

[21] J. A. del Peral-Rosado, J. A. Lopez-Salcedo, G. Seco-Granados, F. Zanier, and M. Crisci, "Achievable localization accuracy of the positioning reference signal of 3GPP LTE," in Proceedings of the 2012 International Conference on Localization and GNSS, pp. $1-6,2012$.

[22] 3GPP, TR 36133, Requirement for Support of Radio Resource Management, Version 11.3.0, 3GPP, 2012.

[23] T. Zhang, D. Xiao, J. Cui, and X. Luo, "A novel OTDOA positioning scheme in heterogeneous LTE-advanced systems," in Proceedings of the 3rd IEEE International Conference on Network Infrastructure and Digital Content (IC-NIDC '12), pp. 106-110, September 2012.

[24] A. A. D'Amico, U. Mengali, and L. Taponecco, "TOA estimation with the IEEE 802.15.4a standard," IEEE Transactions on Wireless Communications, vol. 9, no. 7, pp. 2238-2247, 2010.

[25] Y. Chen, L. Cui, and C. Qi, "Performance analysis of pilot aided channel estimation methods for MBSFN in LTE system," in Proceedings of the International Conference on Computational Problem-Solving (ICCP '11), pp. 682-687, October 2011. 

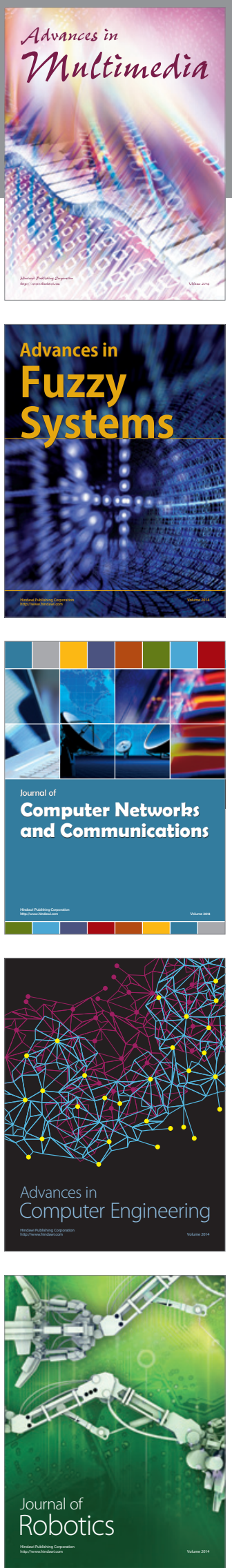

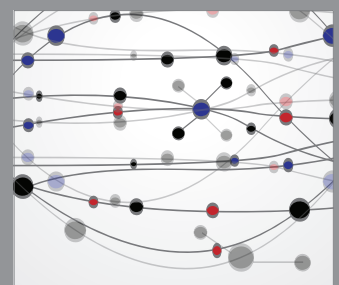

The Scientific World Journal
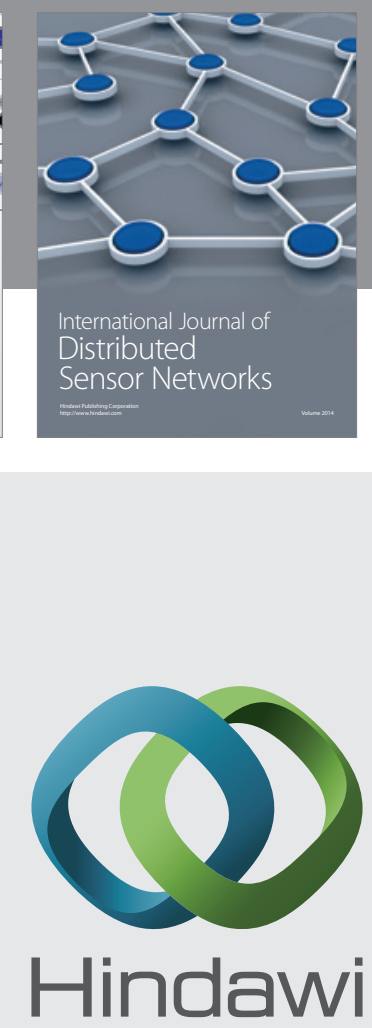

Submit your manuscripts at

http://www.hindawi.com
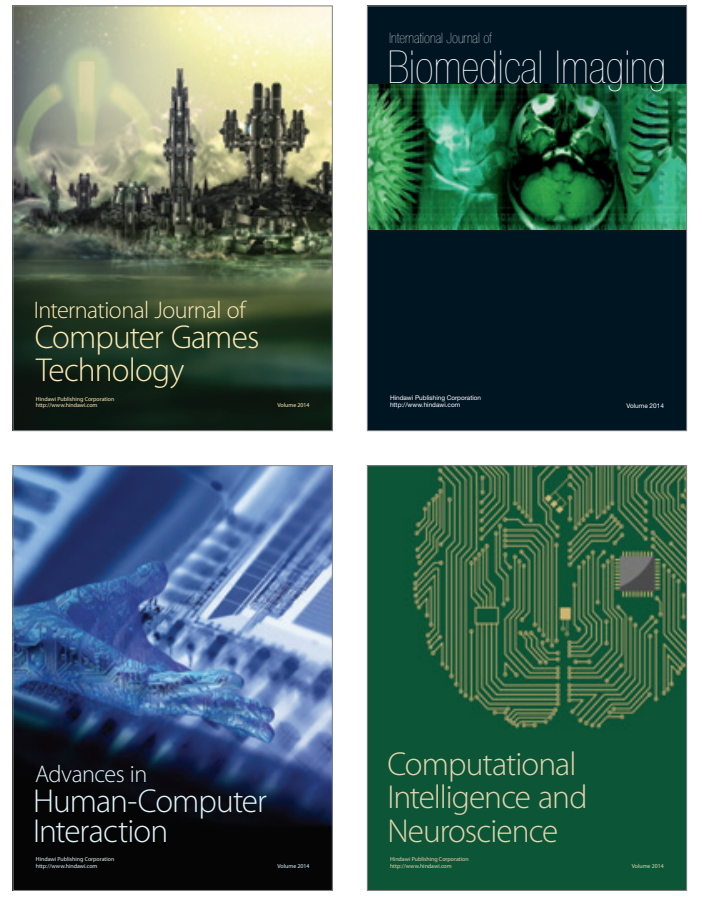
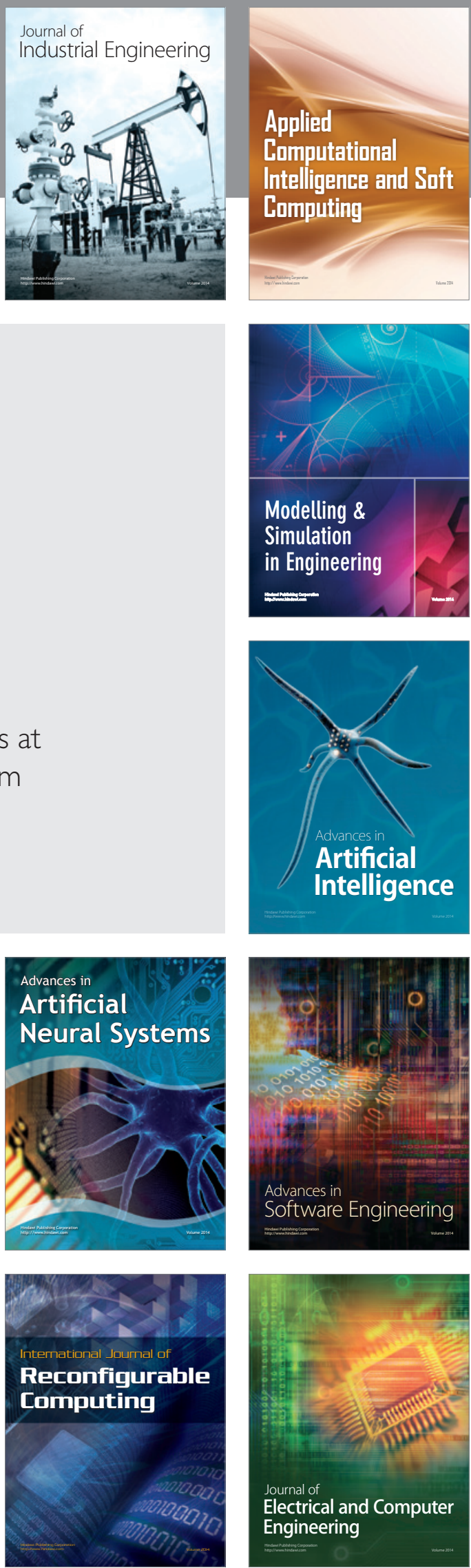